\title{
Hypsarrhythmia and spasms resolution after Valproic acid discontinuation in an infantile spasm patient
}

Mohammed S. Alsallumi, MBBS, SB-PN, SFEE.

\begin{abstract}

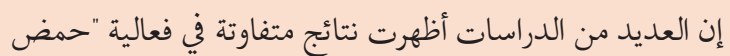

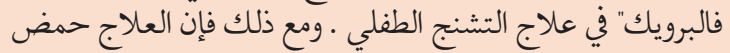

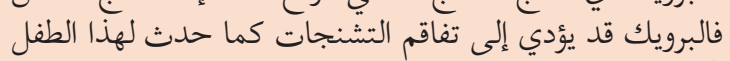

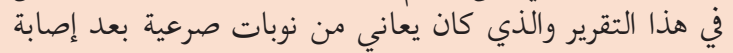

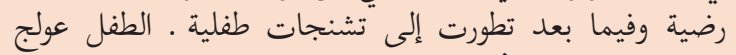

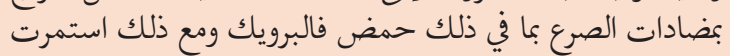

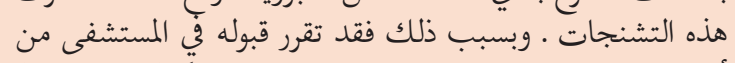

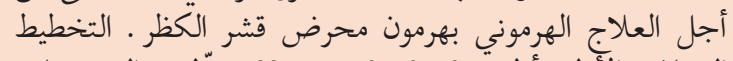
الدماغي الآولي أظهرِ

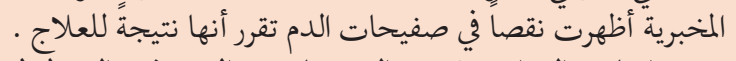

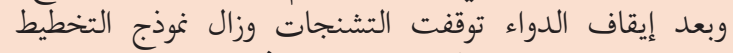
Hypsarrhythmia

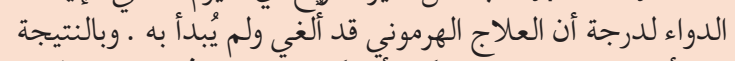

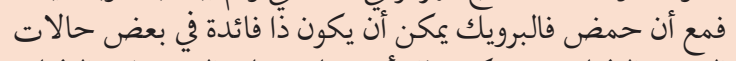

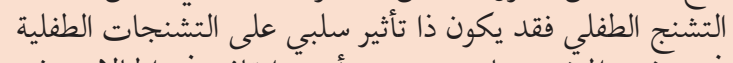

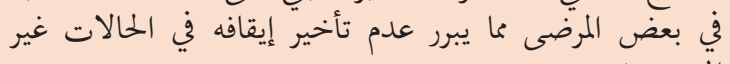

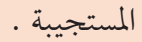

Several studies have reported a variable benefit of valproic acid for the treatment of infantile spasm. However, valproic acid can also worsen spasms, as occurred with this child who presented with posttraumatic seizure which evolved to spasms. The child was started on antiepileptic medications, including valproic acid, despite that spasms persisting. For this reason, she was admitted for adrenocorticotropic hormone therapy. The baseline electroencephalogram showed modified hypsarrhythmia, and the laboratory workup showed thrombocytopenia, which was attributed to the valproic acid. After the valproic acid cessation, the spasms and the hypsarrhythmic pattern resolved dramatically next day, and the intended adrenocorticotropic hormone therapy was not started. Eight months later, she was still free of spasms. In conclusion, though valproic acid might have a beneficial effect in some patients with infantile spasm, it might have a negative impact on spasms in some patients which warrants its discontinuation sooner than later during spasms treatment.
Neurosciences 2019; Vol. 24 (4): 311-314 doi: 10.17712/nsj.2019.4.20190026

From King Abdulaziz Medical City (Algahtani), King Abdullah International Medical Research Center (Shirah), King Saud bin Abdulaziz University for Health Sciences, Jeddah, Kingdom of Saudi Arabia.

Received 23rd March 2019. Accepted 20th June 2019.

Address correspondence and reprint request to: Dr. Mohammed S. Alsallumi, Department of Pediatrics, College of Medicine, Qassim University. E-mail: Drsallumi@gmail.com

ORCID ID: https://orcid.org/0000-0001-8798-3895
Thfan nfantile spasm was first described in 1841 by James West in his own son. ${ }^{1}$ It is an age-specific epileptic disorder that mostly affects infants aged between 4 to 8 months, and the majority present before the age of 24 months. Classically, the spasms are characterized by bilateral, symmetric contraction of the axial muscles lasting for less than 2 seconds followed by less intense tonic contraction lasting between 2-10 seconds. The spasms occur in clusters separated by intervals of 5-30 second. ${ }^{2}$ As they are mostly occurring in infants with developmental delay, infants with infantile spasms are inevitably experience regression in their development and demonstrate a characteristic pattern of hypsarrhythmia on electroencephalogram (EEG). ${ }^{3}$

The spasms are usually refractory to most of the conventional antiepileptic medications, and are usually associated with poor long-term prognosis. ${ }^{3,4}$ Although the treatment guidelines for infantile spasm are limited, hormonal therapy and vigabatrin have higher evidence of effectiveness in the treatment of infantile spasms. ${ }^{5}$ Other medications including topiramate, zonisamide, valproic acid, pyridoxine and ketogenic diet might

Disclosure. The authors declare no conflicting interests, support or funding from any drug company. 
help to reduce spasms frequency and are often used after ACTH and vigabatrin. ${ }^{2,3}$ Although the valproic acid produced benefits in spasms reduction, ${ }^{6} \mathrm{I}$ am reporting this interesting case in which the valproic acid resulted in the perpetuation of the clinical spasms and electrographic hypsarrhythmia in a child with refractory epilepsy secondary to post-traumatic injury. Immediately after discontinuation of the drug, spasms and hypsarrhythmia resolved completely.

Case Report. Patient's information. A 24 months old girl a case of global developmental delay and symptomatic epilepsy secondary to severe traumatic brain injury due to a vehicle traffic accident at the age of 3 months. At that time, she was admitted and had several surgeries including a ventriculoperitoneal shunt for post hemorrhagic hydrocephalus. Post-discharge from the hospital, she continued to have unprovoked partial and generalized seizures. This evolved into spasms in the form of the whole body stiffening with flexion of arms and head deviation to the right side. Her eyes would be staring ahead during the event. She had these fits in 4-5 clusters per day, and these were more prominent upon awakening from sleep. She was initially treated with phenobarbital for the partial and generalized seizures, but when spasms developed, she was given valproic acid since the age of 4 months.

According to history, there was no clear change in the frequency of spasms after starting the valproic acid, however, no documentation from previous reports. Vigabatrin was given for 6 months without response. There was no clear documentation of EEG at the time of diagnosis nor during the course of illness. She was also prescribed levetiracetam without success. She was referred to our hospital for further management.

Clinical findings. On examination, she is markedly failing to thrive. She has Spasticity in all her limbs, deep tendon reflexes were exaggerated with clonus and planter reflex was upgoing. She is weaker in the left side in comparison to the right side. Systemic examination was unremarkable.

Diagnostic assessment. she was admitted at age of 24 months for ACTH therapy. A baseline EEG was carried out which showed modified hypsarrhythmia due to asymmetry in the form of hemihypsarrythemia (Figure 1).

During the admission, she was found to have thrombocytopenia, at which point the valproic acid was discontinued. On the day after valproic acid was discontinued, the spasms stopped, although ACTH was not given. Repeat EEG 1 week later showed focal slowing over the right side with the resolution of the hypsarrhthmic pattern (Figure 2).

Therapeutic intervention. because of the dramatic improvement of the spasms after valproic acid discontinuation, no therapeutic intervention was

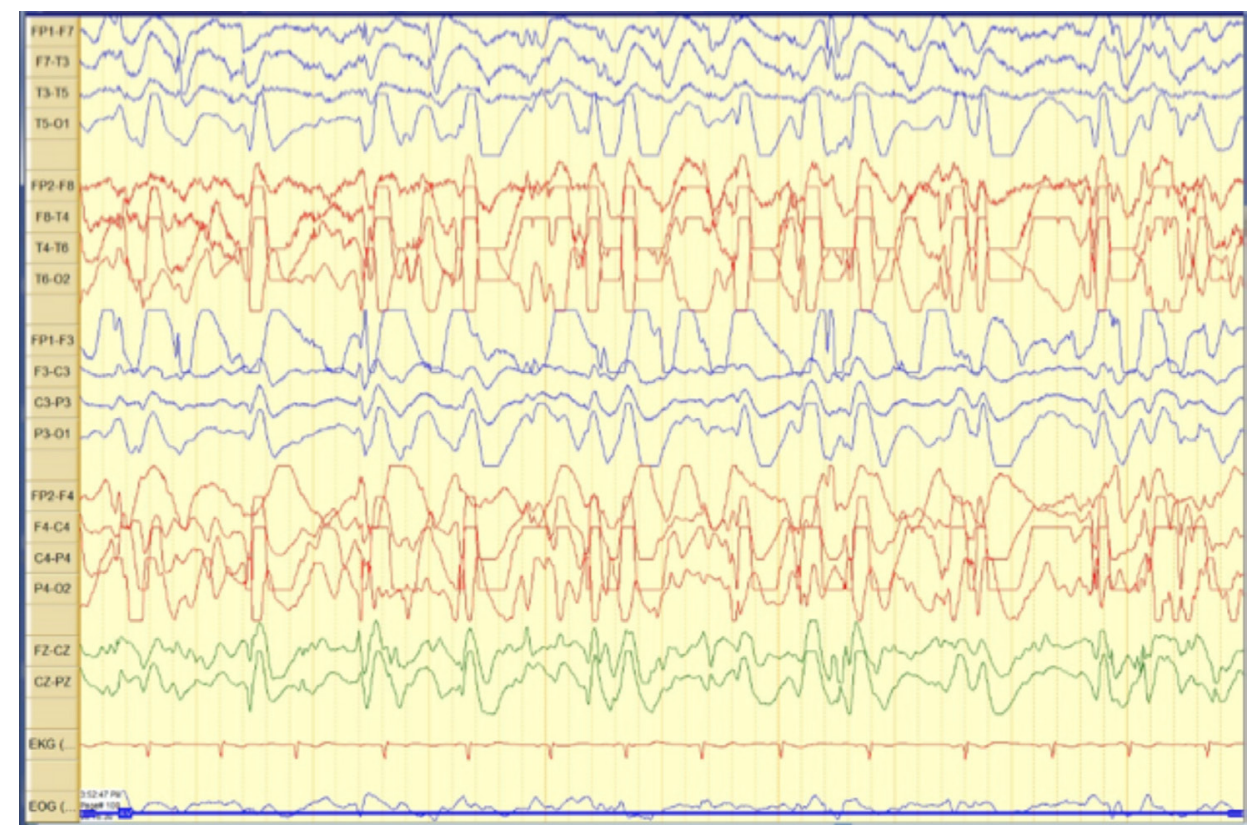

Figure 1 - The baseline EEG, (Bipolar montage, sensitivity: $7 \mathrm{mV} / \mathrm{mm}, \mathrm{HF} ; 70 \mathrm{~Hz}, \mathrm{LF}: 1 \mathrm{~Hz}$ ) shows a disorganized, chaotic activity intermixed with high amplitude, multifocal spike and slow wave discharges in the right hemisphere, consistent with "Modified hypsarrhythmia". 


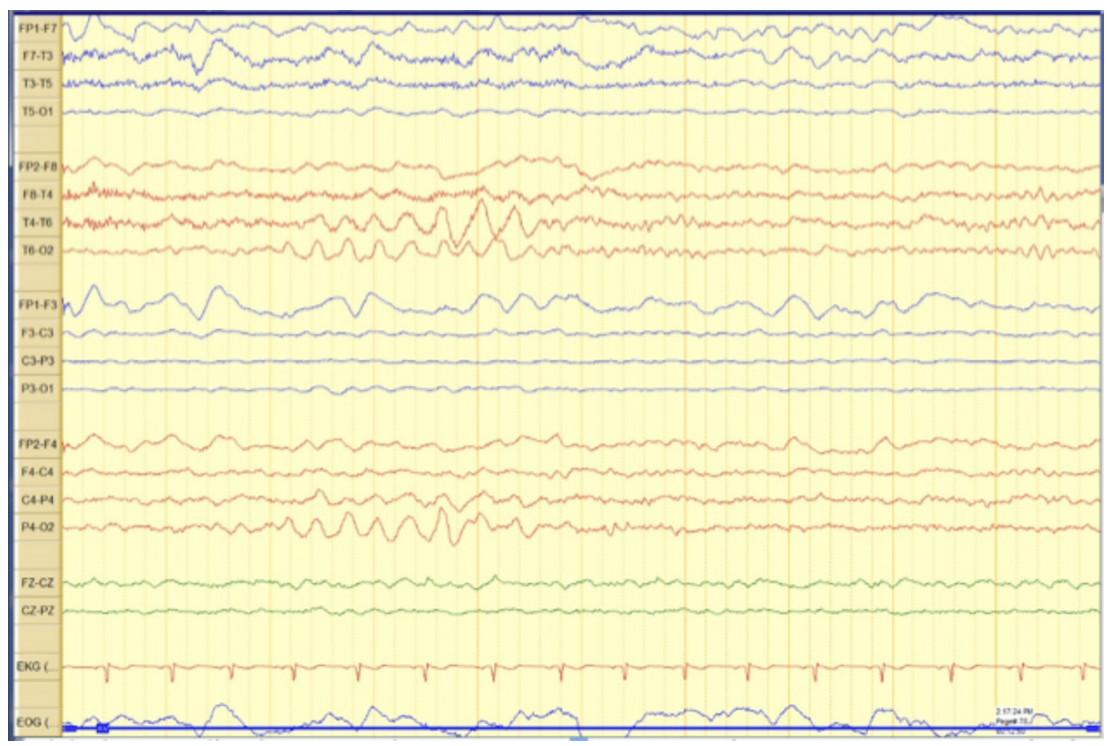

Figure 2 - Electroencephalogram after valproic acid discontinuation, (Bipolar montage, sensitivity: $7 \mathrm{mV} / \mathrm{mm}, \mathrm{HF}$; $70 \mathrm{~Hz}$, LF: $1 \mathrm{~Hz}$ ) shows disappearance of the modified hypsarrhythmic pattern.

provided for the patient.

Follow up and outcomes. The child was discharged home on no antiepileptic drugs and remained seizure free for 8 months post discharge. However, she developed focal seizures, which were treated with

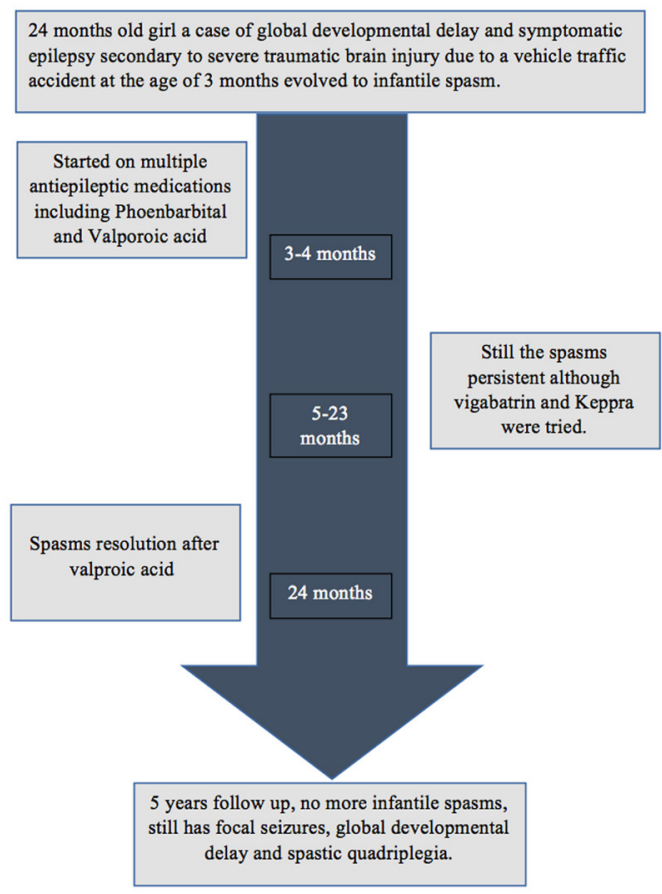

Figure 3 - Timeline for the chronological sequence of the patient's presentation. topiramate, with partial response. At the age of 5 years, she was still having focal seizures, and her development was severely delayed with spastic quadriplegia (Figure 3).

Discussion. Valproic acid is a broad-spectrum antiepileptic drug that is used for multiple types of epilepsy. Although there are no controlled trials supporting the use of valproic acid in the infantile spasm, the reported benefit of valproic acid in the infantile spasm patients is variable, with the degree of spasms reduction, ranging between $40 \%$ to $70 \%$ among patients who do not respond to ACTH. ${ }^{6}$ In another prospective study, 91 children were started on valproate, $39.5 \%$ of them showed a good response, but seven later relapsed while on the same dose of valproate. ${ }^{7}$

In this case, the patient had a post traumatic partial and generalized seizures which evolved to the spasms semiology. Valproic acid was started and despite that, the spasms persisted. Once the valproic acid was stopped, the clinical spasms and the hypsarrhythmia pattern in the EEG resolved dramatically. Opposite to previous reports, in this case, the valproic acid acted as an exacerbating or emerging factor for her spasms. It is possible that resolution of spasms is a spontaneous remission due to increasing age of the patient, however, it is plausible to claim that it is related to discontinuation of VPA for the following 2 reasons; first, the spasms in this patient is symptomatic secondary to old traumatic brain injury which makes the spontaneous resolution less likely and, second, the time lock of cessation of 
spasms immediately post discontinuation of the drug.

During the literature review for infantile spasms exacerbated by valproic acid, I identified one reported case by Itonaga et al. ${ }^{8}$ In this case, the spasms were exacerbated after the valproic acid administration. As per the authors, it induced a novel complex partial seizure, suggesting that the patient had distinctive clinical seizures due to non-ketotic hyperglycinemia.

In addition to the clinical spasms exacerbation, other adverse effects could occur when the valproic acid is introduced to the infantile spasm patients. Sivathanu et al. ${ }^{9}$ reported an infant with infantile spasm that started on sodium valproate, clonazepam and ACTH. After a period of improvement, she developed signs of encephalopathy including vomiting, altered level of sensorium and increased the seizures frequency which was dramatically improved after the valproic acid stopped. The encephalopathy was attributed to the valproate-induced hyperammonaemia or hepatic encephalopathy.

Unfortunately, the ammonia level in our patient was not ordered when the patient was receiving valproic acid which could cause hyperammonemia. However, such induced hyperammonemia is usually associated with encephalopathic clinical features rather than the spasms emergence or aggravation. The epilepsy etiology for the patient is post traumatic which had evolved later to the spasms. However, the reason of the dramatic spasms resolution after the valproic cessation remains unclear.

In conclusion, though valproic acid might have a beneficial effect in some patients with infantile spasm, a negative impact on spasms might also be encountered, which warrants discontinuation of this drug sooner than later during spasms treatment.
Acknowledgments. I want to specially thank $D r$. Muhammed T. Alrifai, for his kind support and motivation to report this case. Also, I want to thank Dr. Sameer A. Almehmadi for his appreciated cooperation during the preparation of this case report, and American Manuscript Editors (www.americanmanuscripteditors. com) for English language editing.

\section{References}

1. West W. On a particular form of infantile convulsions. Lancet 1952; 1: 724-725.

2. Swaiman K, Ashwal S, Ferriero D, Schor N, Finkel R, Gropman, A, et al. Swaiman's pediatric neurology. Edinburgh (UK): Elsevier; 2018.

3. Taghdiri MM, Nemati H. Infantile spasm: a review article. Iran J Child Neurol 2014; 8: 1-5.

4. Hancock E, Osborne JP, Milner P. Treatment of infantile spasms. Cochrane Database Syst Rev 2002; 2: CD001770.

5. GR Nelson. Management of infantile spasms. Transl Pediatr 2015; 4: 260-270.

6. Glaze, DG, editor. Management and prognosis of infantile spasm. In: Nordli DR, Dashe JF, editors. Uptodate. [ cited 2018; Retrieved March 2019]. from: https://www.uptodate. com/contents/management-and-prognosis-of-infantile-spasms

7. Chandra S, Bhave A, Bhargava R, Kumar C, Kumar R. West Syndrome: Response to valproate. Front Neurol 2012; 3: 166.

8. Itonaga T, Okanari K, Korematsu S, Kure S, Izumi T. Valproic acid exacerbated infantile spasms and induced novel complex partial seizures in an infant with non-ketotic hyperglycinemia. Epilepsy \& Seizure 2014; 7: 30-36.

9. Sivathanu S, Sampath S, Veerasamy M, Sunderkumar S. Encephalopathy in an infant with infantile spasms: possible role of valproate toxicity. BMJ Case Rep 2014; 2014. 